\title{
Dynamical continuum simulation of condensed matter from first principles
}

\author{
Oliver Strickson, ${ }^{*}$ Nikos Nikiforakis, and Emilio Artacho $\odot$ \\ Cavendish Laboratory, University of Cambridge, J. J. Thomson Avenue, Cambridge CB3 OHE, United Kingdom
}

(Received 23 May 2019; revised manuscript received 22 October 2019; published 24 December 2019)

\begin{abstract}
Macroscale continuum mechanics simulations rely on material properties stemming from the microscale, which are normally described using predetermined phenomenological equations of state (EOS), which depend on physical parameters usually obtained from experiments. A method is proposed for the automatic generation of first-principles unconstrained EOSs using a Gaussian process on a set of $a b$ initio molecular dynamics simulations, thereby closing the continuum equations with the accuracy determined by the underlying firstprinciples theory. The method is validated for bulk silicon using density-functional theory (DFT) by comparing planar (one-dimensional) shock waves with experiment, and also with an explicit first-principles molecular dynamics simulation, both in the hyperelastic regime. Its applicability is then illustrated it for two-dimensional shock waves in DFT bulk silicon, following their nonlinear, temperature-dependent, anisotropic dynamics after a cylindrical region is instantaneously heated. The method is applicable to condensed matter systems in other regimes, using well-known continuum techniques and/or molecular dynamics simulations at larger scales.
\end{abstract}

DOI: 10.1103/PhysRevResearch.1.033199

\section{INTRODUCTION}

Continuum mechanics simulations are of great importance for the simulation of macroscopic condensed matter, from flows in the oil and gas industry [1,2] or the modeling of high strain-rate structural deformation [3], to shock waves and detonation in condensed-phase media [4-6]. The phenomena these simulations describe have their origin in the interactions and dynamics of electrons and nuclei at a much smaller scale. Continuum mechanical theories disregard the constituent particles, however, using phenomenological equations of state (EOS) [7], to which a substantial research effort is dedicated (see, e.g., Refs. [8-13]). Electronic structure methods allow the accurate and predictive evaluation of the EOS of condensed matter from first principles, and it has been done for either cold or isotropic materials [14-16]. The general situation is usually much more complex, however. For solid materials, the EOS is a function of deformations beyond volume change if it is to capture the behavior of a solid under shear loading, and effects beyond linear elasticity mean the use of elastic constants would be insufficient. To the strain dependence, temperature adds an extra dimension to the effective parameter space, totalling seven in the case of a general, anisotropic solid.

Here we describe a procedure for computing an EOS from first principles in a general way, that neither depends on the material nor imposes any functional form and parameter

\footnotetext{
*Current address: The Alan Turing Institute, British Library, 96 Euston Road, London NW1 2DB; ostrickson@turing.ac.uk

Published by the American Physical Society under the terms of the Creative Commons Attribution 4.0 International license. Further distribution of this work must maintain attribution to the author(s) and the published article's title, journal citation, and DOI.
}

fitting, in contrasts to traditional EOSs, such as MieGrüneisen ones $[7,17]$. The generality and accuracy of the former is only limited by those of the underlying first-principles theory, although the latter keeps an efficiency advantage.

Our EOS is constructed using a machine-learning Gaussian process that probes the relevant space by a series of ab initio molecular-dynamics (AIMD) simulations. We illustrate the method with DFT for hyperelastic solids, but the procedure is applicable much more generally in systems and underlying theory. Figure 1 shows results for anisotropic shock waves emanating into bulk silicon using this method, after a cylinder was suddenly heated, described in more detail below.

\section{METHOD}

\section{A. Continuum simulations}

For the continuum simulations, the numerical solution of a system of conservation laws is obtained in the Eulerian frame [18]. A general deformation is represented by the deformation gradient tensor, $\boldsymbol{F}=\partial \boldsymbol{x} / \partial \boldsymbol{X}$, where $\boldsymbol{X}$ is a material point in the undeformed configuration, and $\boldsymbol{x}$ is its displaced position. The system evolves in time according to [18]

$$
\begin{gathered}
\left(\rho F_{i j}\right)_{t}+\left(\rho F_{i j} u_{k}-\rho F_{k j} u_{i}\right)_{k}=0, \\
\left(\rho u_{i}\right)_{t}+\left(\rho u_{i} u_{j}-\sigma_{i k}\right)_{k}=0, \\
(\rho E)_{t}+\left(\rho u_{k} E-u_{i} \sigma_{i k}\right)_{k}=0,
\end{gathered}
$$

with $\rho$ being the mass density, $\boldsymbol{u}$ the velocity field, $E$ the internal energy, and $\sigma$ the Cauchy stress, with the initial constraint

$$
\nabla \times \boldsymbol{F}_{i}=0 .
$$

An EOS closes the system giving the internal energy for any deformation. For the symmetric strain (excluding rotations), 


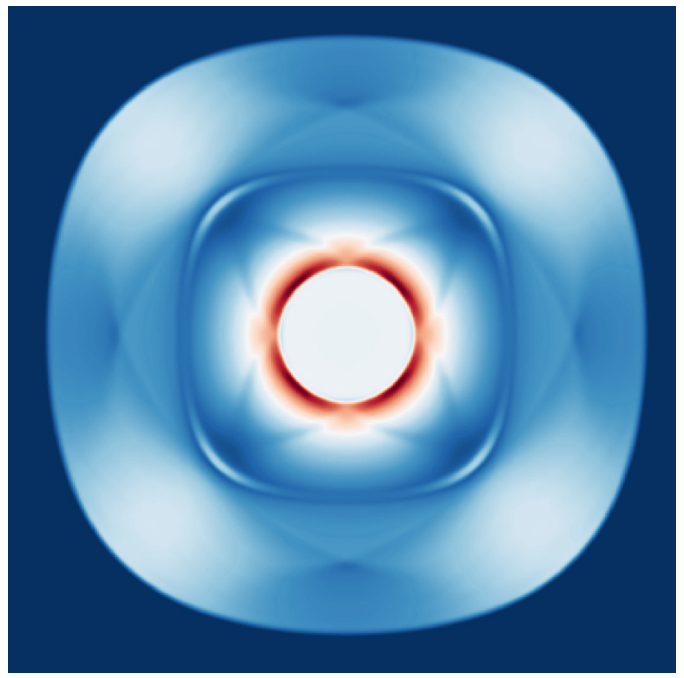

FIG. 1. Deviatoric stress from first principles for a shock wave in bulk silicon at zero pressure and $40 \mathrm{~K}$ at time $t=R / \alpha\left(\alpha=10^{4} \mathrm{~m}\right.$ $\mathrm{s}^{-1}$ ) after the sudden heating of a macroscopic cylindrical region of radius $R$ (white circle). Dark blue (red) indicates $0 \mathrm{MPa}(285 \mathrm{MPa}$ ) as lowest (highest) value. The inner cylinder is at $136 \mathrm{MPa}$.

we use the right Cauchy-Green tensor,

$$
\boldsymbol{G}=\boldsymbol{F}^{\top} \boldsymbol{F} .
$$

The Cauchy stress $\boldsymbol{\sigma}$ is given in terms of $\boldsymbol{G}$ as

$$
\sigma=2 \rho F_{i l}\left(\frac{\partial E}{\partial G_{l m}}\right)_{S} F_{j m},
$$

where the derivative for stress is at constant entropy: this would suggest $E(\boldsymbol{G}, S)$ as convenient form of EOS. There is, however, no need for obtaining entropy values, which is expensive for AIMD. The calculation of strain derivatives of the energy for (different values of) constant entropy is what is needed. Instead, we can define the reference tag $E_{0}$ as the internal energy (nuclear kinetic energy plus total electronic energy) if it were adiabatically brought to the reference configuration from the deformed one. As defined, the mapping between $E_{0}$ and entropy does not depend on deformation, and, therefore, $E_{0}$ can be used to label the isentropes. The EOS is expressed then as $E\left(\boldsymbol{G}, E_{0}\right)$, and one obtains $(\partial E / \partial \boldsymbol{G})_{S}$ from an entropy preserving (thermally isolated) quasistatic AIMD simulation for a given $E_{0}$. That is, we follow isentropes without needing to know the value of $S$.

The numerical solution to Eqs. (1)-(3) has been extensively discussed [18-21]. A finite-volume formulation is used here, with fluxes from the FORCE scheme (see Chap. 7 in Ref. [22]) using the MPWENO-5 reconstruction [23]. The finite-volume formulation in the Eulerian frame allows capturing correct weak solutions (shock waves). The particular nonlinear elasticity formulation and solution method used here illustrate the utility of our EOS, but the same ideas can be readily ported to other situations.

\section{B. First-principles molecular dynamics}

The EOS used there was obtained from first-principles simulations of silicon based on DFT. AIMD simulations were performed with the SIESTA method [24], using the PBE [25] exchange-correlation functional. The basis functions for the valence electrons, and the pseudopotential for the $\mathrm{Si}$ core electrons are as described in Ref. [26]. The mesh used for real-space integrals was well converged with a 100-Ry cutoff. The simulations in this paper are for (deformed and heated) bulk $\mathrm{Si}$ in the diamond crystal structure, and a $2 \times 2 \times 2$ supercell of the eight-atom conventional unit cell was used for the purpose, unless otherwise stated. It was then dynamically deformed following AIMD isentropes as defined in the next section, depending on the particular point sampled in the seven-dimensional parameter space. A $2^{3}$ grid of $\mathbf{k}$ points was used on the 64-atom simulations, giving an effective cutoff length of $11 \AA$ [27], except for the explicit AIMD shock simulations, for which the sampling was chosen so as to be equivalent to the $\Gamma$ point sampling of the 960 -atom shock simulation described below. Thermal electronic contributions being small, $300 \mathrm{~K}$ was used throughout as electronic temperature.

Verlet integration (modified as described below) was used to follow isentropes, with $1 \mathrm{fs}$ timesteps. 480 separate deformations were performed, with 2-ps AIMD runs. For each deformation, an initial configuration was obtained by equilibrating the system using the Tersoff empirical potential [28] on the intended undeformed state, before switching to DFT, which was further integrated for $250 \mathrm{fs}$ before starting the deformation, and for $250 \mathrm{fs}$ after finishing it, in order to obtain averaged final quantities.

\section{Isentropes and equation of state}

States along an isentrope are extracted directly with molecular dynamics in a slowly deforming box. An alternative was suggested by Chentsov and Levashov [29] for liquids, using a sampling in density and temperature before solving an ODE to find $E(\rho, S)$. In our direct procedure, AIMD gives an isentropically deformed state to a given target deformation, starting from an undeformed reference state at a given (randomly sampled) $E_{0}$ value, obtained by equilibrating to a given temperature. The box is steadily deformed by slowly varying the box vectors in a linear process from undeformed to the deformed target. The entropy change is made arbitrarily small by decreasing their rate of variation, since a slowly varied parameter of a Hamiltonian preserves entropy to first order in this parameter variation rate [30]. To demonstrate that we can follow an isentrope numerically, we show that the process is adiabatic and reversible. That is

$$
d E \rightarrow \frac{1}{2} V \operatorname{tr}\left(\boldsymbol{\sigma}^{\top} \mathrm{d} \boldsymbol{G}\right)
$$

as the time derivative of the deformation vanishes. Additionally, if the process is reversed, the energy difference between the initial and final states tends to zero. The quantities involved in this expression are equilibrium ensemble averages.

Figure 2(a) shows both the difference in energy from this process and the integrated work. For an isentropic process, both should be zero, any difference representing systematic error introduced by the process. Two cases are illustrated: a uniaxial elastic compression of 0.9 relative volume (representative of the deformations we consider), and an uniaxial compression of a liquid, to 0.8 relative volume. The latter 

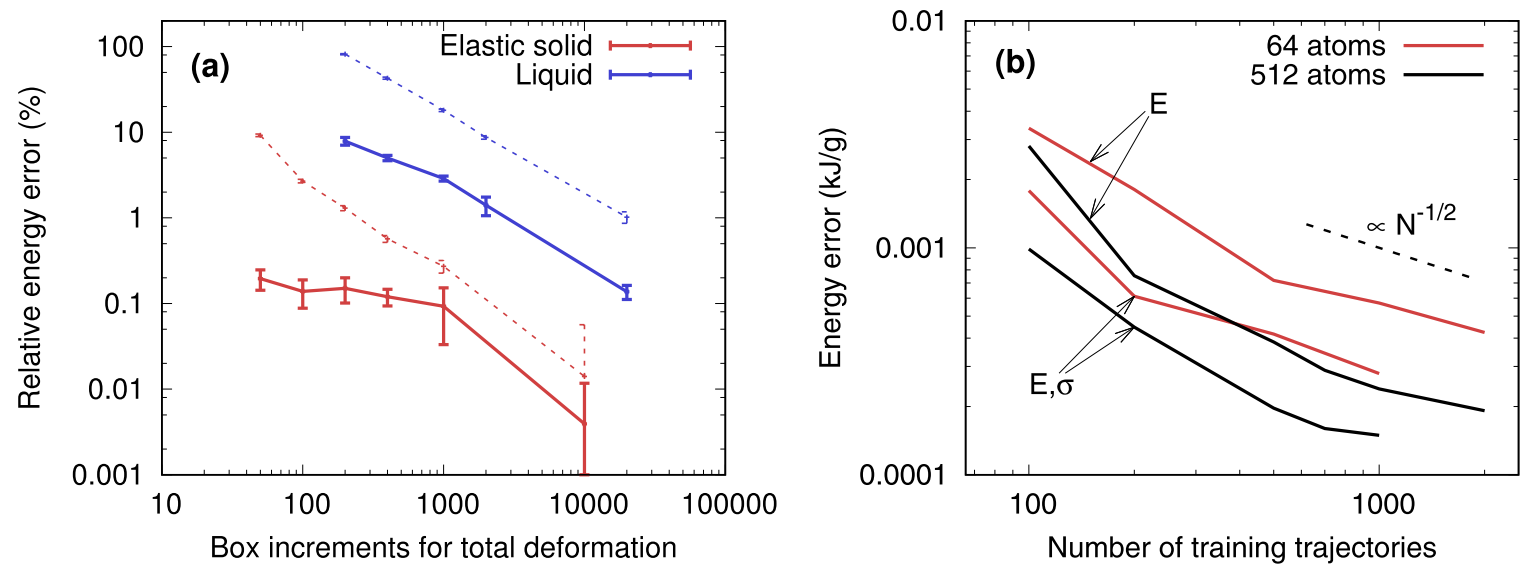

FIG. 2. Sources of error. (a) Energy convergence for isentropic trajectories: from the work done by the deforming box (solid lines) and from the internal energy (dotted lines). Red (blue) lines: crystalline (liquid) silicon deformed to a uniaxial strain of 0.9 (0.8). Points and error bars indicate ensemble averages sampling equivalent trajectories. (b) Reconstruction error from the Gaussian process versus number of isentropic deformation trajectories, training from energy alone ("E") or energy and stress ("E, $\sigma$ ").

case is more challenging since it takes additional time for the fluid to relax to hydrostatic stress. We can therefore apply deformations to stresses of tens of GPa over $\sim 1$ ps on 64 -atom cells and achieve relative errors in the straining energy of around $1 \%$, and of $0.2 \%$ when computed by integrating the work.

\section{Gaussian process}

Figure 3 shows slices through the silicon EOS used in Fig. 1. From AIMD we have a discrete sampling of the energy surface. The points must be interpolated to evaluate the energy of any deformation at any temperature. A suitable interpolation method and a sampling procedure are crucial components of this scheme. We use Gaussian process regression for the interpolation [31,32] for several reasons. First, its ability to handle multidimensional data. Second, the interpolated function is smooth (with a suitable covariance function): we require the interpolant to have continuous second derivatives, since these affect wave speeds, thereby avoiding unphysical

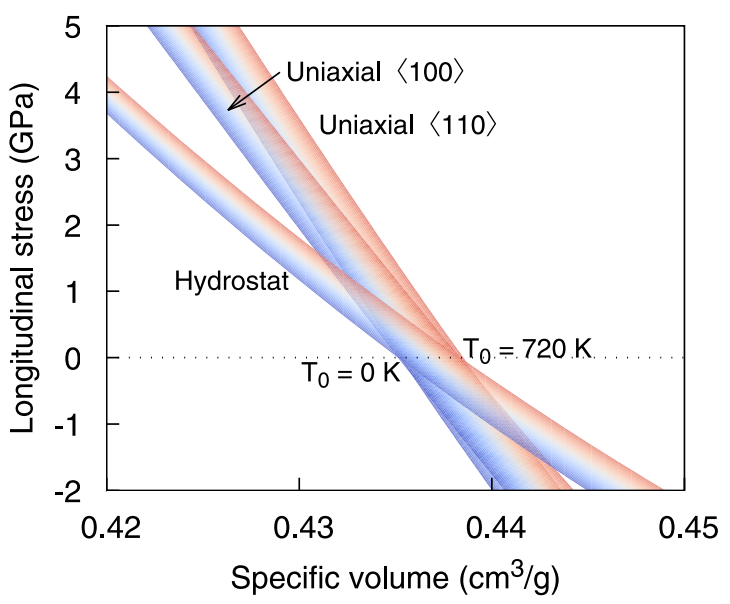

FIG. 3. Compressive isentropes from the first-principles EOS for silicon. Each compression is shown for a range of isentropes, colored by their initial temperature. wave splitting. Third, it can incorporate derivate observations (e.g., from pressure) into the learning process and predict derivatives of the interpolated function (and therefore pressures).

The Gaussian process prediction takes the form

$$
\hat{t}^{\prime}=\boldsymbol{k}^{\top} \boldsymbol{C}^{-1} \boldsymbol{t},
$$

where $\boldsymbol{t}$ is the vector of observed values (total energies and their derivatives with respect to $\boldsymbol{G}$ ) and $\boldsymbol{C}$ is the covariance matrix, computed from the training data as

$$
C_{i j}=C\left(\boldsymbol{x}^{(i)}, \boldsymbol{x}^{(j)}\right)+v^{2} \delta_{i j},
$$

where $\boldsymbol{x}=\left(\boldsymbol{G}, E_{0}\right)$ is the vector of inputs, and with the squared exponential covariance function between energy observations,

$$
\begin{aligned}
& C\left(\boldsymbol{x}^{(1)}, \boldsymbol{x}^{(2)}\right) \\
& \quad=\zeta^{2} \exp \left\{-\sum_{i, j} \frac{\left[G_{i j}^{(1)}-G_{i j}^{(2)}\right]^{2}}{2 r_{G_{i j}}^{2}}-\frac{\left[E_{0}^{(1)}-E_{0}^{(2)}\right]^{2}}{2 r_{E_{0}}^{2}}\right\} .
\end{aligned}
$$

The vector $\boldsymbol{k}$ contains the covariances of the input to predict, $\boldsymbol{x}^{*}$, with each of the observations; that is,

$$
k_{i}=C\left(x^{(i)}, x^{*}\right) .
$$

Covariances between value and derivative observations, and between two derivative observations, are the corresponding derivatives of the $C\left(\boldsymbol{x}^{(1)}, \boldsymbol{x}^{(2)}\right)$ function.

The interpretation of the hyperparameters in Eq. (10) is as follows: $\zeta$ sets the scale of the inferred function, $v$ represents position-independent Gaussian noise in the outcomes that is independent of the inputs, and $r_{G_{i j}}$ is the length scale over which the function varies with $G_{i j}$. Separate noise hyperparameters are used for value and derivative observations.

The sampling is performed by choosing $\boldsymbol{G}$ uniformly at random over a problem-specific domain of interest, before converting it to a deformation gradient $\boldsymbol{F}$ (by a Cholesky decomposition), and thence to a target lattice $\boldsymbol{F} \boldsymbol{L}$, where $\boldsymbol{L}$ 
is the matrix whose columns are the lattice vectors. For larger dimensionality other samplings may be more suitable [35].

The sampling domain can be chosen generously to include the range over which the deviatoric part of the strain is expected to be less than or equal to the yield criterion, according to, for example, a continuum plasticity model, and with the isotropic part of the strain less than some bound. For the EOS obtained here, we sample each component independently uniformly over the range $[0.9,1.1]$ for the diagonal components, and $[-0.3,0.3]$ for the off-diagonal ones. $E_{0}$ is sampled by varying the initial $T_{i} \in[0,800] \mathrm{K}$. Since $E_{0}$ is the dominant contribution to the internal energy, the fitting is improved by defining $E^{\prime}$

$$
E^{\prime}\left(\boldsymbol{G}, E_{0}\right)=E\left(\boldsymbol{G}, E_{0}\right)-E_{0}
$$

as the quantity to interpolate.

The error from the reconstruction is shown in Fig. 2(b), from an EOS computed using an empirical potential, allowing larger sampling. For the databases where gradient information is used, all six components of the gradient are included for one-sixth of the points in the database. The figure shows that this is always beneficial, but much more so for small databases, where it can reduce the error by a factor of four. In addition, if symmetry is exploited, the sampling efficiency is increased by a factor that depends on the crystal system (eight for cubic).

\section{RESULTS AND VALIDATION}

Validation of the multiscale method proposed here is provided by the comparison shown in Fig. 4 for properties of silicon shocked with a flat two-dimensional perturbation, giving rise to a flat shock wave propagating in one dimension, following Hugoniot relations. In particular, the longitudinal stress versus volume and the shock velocity versus particle velocity are displayed for hyperelastic shock propagation along three different main directions in the cubic solid. The results satisfactorily compare with experiments [33,34] as well as with independent simulations for the same DFT silicon obtained from an ab initio Hugoniot calculation [26].

In addition, a full, explicit first-principles shock wave was simulated using AIMD with the same DFT as used here. A $2 \times 3 \times 20$ supercell with $960 \mathrm{Si}$ atoms representing an 80 -layer-thick slab was pushed with a piston along the (001) direction with a velocity of $360 \mathrm{~m} / \mathrm{s}$. The $k$-point sampling used for this simulation was reduced to the $\Gamma$ point for computational reasons. The system was first equilibrated to room temperature and zero pressure with the Tersoff potential for several ps, and then with DFT AIMD (after rescaling to readjust for the slightly different equilibrium lattice parameters of both theories) for an additional $0.5 \mathrm{ps}$. The shock wave was then produced by imposing the constant velocity motion of the first surface layers of atoms (one conventional unit cell deep, 48 atoms in total), representing the piston, compressing the rest of the system along the $z$ direction. The particle velocity was chosen to be before the experimental yield, as seen in Fig. 4. The steady shock speed is extracted from the simulations as described in Ref. [36], resulting in $8.39 \pm 0.013 \mathrm{~km} \mathrm{~s}^{-1}$, which is $2 \%$ lower than the value obtained with the first-principles continuum method described
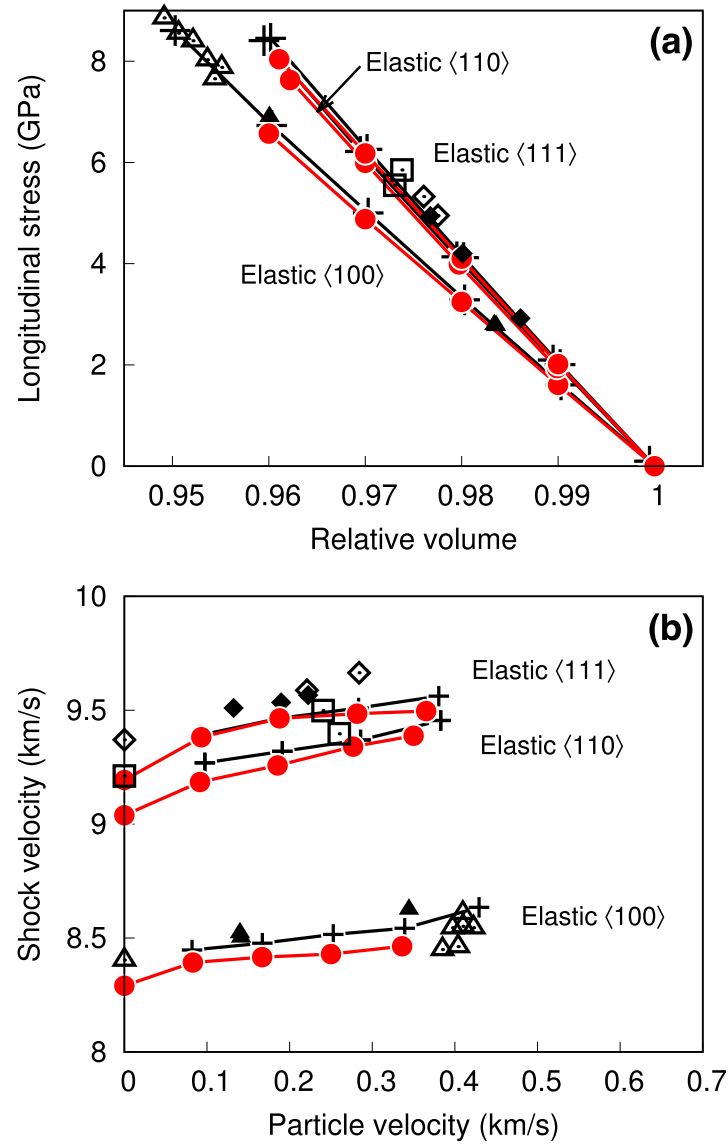

FIG. 4. Validation for shock waves. Hugoniot states for silicon from an initially uncompressed state at $300 \mathrm{~K}$. Red lines $(\bullet)$ : results of this work. Black lines (+): direct atomistic computation of the Hugoniot locus for the same DFT silicon [26]. Experimental results are shown for Refs. [33] (hollow symbols) and [34] (solid): triangles represent shocks along $\langle 100\rangle$, squares for $\langle 110\rangle$, and diamonds for $\langle 111\rangle$.

in this work (with equivalent $k$-point sampling), offering again satisfactory agreement.

It should be remembered, however, that the method described here is of much more general applicability than the planar shock waves, accessible by the method in Ref. [26], which makes explicit use of the Hugoniot relations. Figures 1 and 5 illustrate a much more general case that cannot be simulated otherwise, namely, for a shock wave generated in bulk silicon from the sudden heating to $600 \mathrm{~K}$ of a cylinder of radius $R$ in zero-pressure $40-\mathrm{K}$ bulk silicon. The figures show the behavior of the deviatoric stress (Fig. 1), the radial material velocity [Fig. 5(a)] and the transverse material velocity [Fig. 5(b)] at a time after the initial shock.

The initial cylindrical shock is deformed into the displayed shapes due to the anisotropy of the material. The two main radial wave fronts, namely, the shock and the release waves [outer front and the separation between red and blue in Fig. 5(a), respectively], are only slightly deformed with respect to the cylindrical equivalent features one would find for isotropic materials. Other features are much more subtle, however, such as the petal shapes within the red region of Fig. 5(a), and the boundary separating areas with and without 


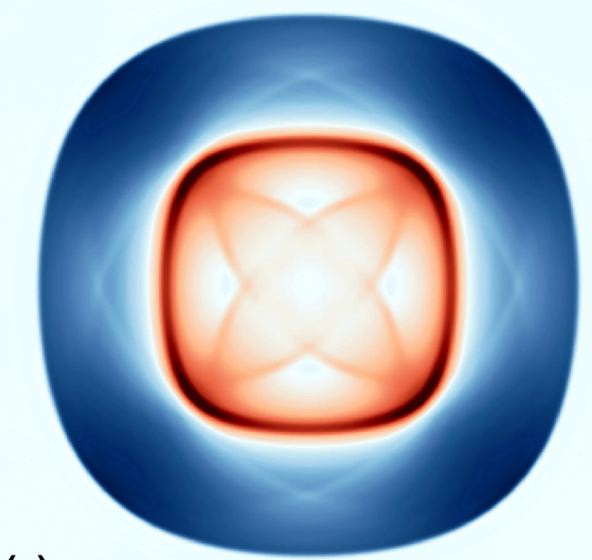

(a)

FIG. 5. Hot cylinder: radial/transverse material velocity (left/right), for a shock wave in silicon from sudden heating as in Fig. 1. Lowest values for radial $(-12.5 \mathrm{~m} / \mathrm{s})$ and transverse $(-1.5 \mathrm{~m} / \mathrm{s})$ appear dark red. Highest respective values $(8.5$ and $1.5 \mathrm{~m} / \mathrm{s})$ in dark blue.

significant transverse material velocity [colored versus white in Fig. 5(b), respectively] within the region between the shock and release waves. Interestingly, this boundary flattens along $\langle 110\rangle$ directions, in contrast with the main fronts, which are flatter along the $\langle 100\rangle$ directions. Note the remarkable behavior of the transverse material velocity along [100] and [010] directions of the silicon crystal (aligned with the $x$ and $y$ axes in the figures, respectively). Special points are apparent for the depicted time frame at which the velocity changes very abruptly, peaking strongly at specific radii, and changing sign tangentially, also very sharply. There is a scale invariance in the continuum equations that allows addressing macroscopic scales, which is out of reach for purely atomistic simulations.

\section{CONCLUSION}

In summary, a two-scale method has been demonstrated for the generation of EOSs for macroscopic continuum simulations of condensed matter based on first-principles molecular dynamics. The AIMD simulations are performed on a sample of points selected by a machine-learning Gaussian process in the space of parameters, for the required EOS to be effectively interpolated to any other point, as requested by the continuum mechanics simulation. As a first step, it has been illustrated on complex hyperelastic shock waves in bulk silicon as obtained from DFT calculations, for which the method has been validated. Condensed matter systems of other forms or in other regimes, such as liquids, glasses, polycrystalline solids, or solids under plastic deformation, would also be amenable to this method or extensions thereof, using continuum techniques (e.g., assuming yield behaviors for plastic deformation [37]) and MD simulations at larger scales [38]. The method described in this paper brings first principles to a wide range of continuum mechanics, including for materials that have not been synthesized yet.

\section{ACKNOWLEDGMENTS}

We are grateful for the support of Alan Minchinton. O.S. acknowledges funding from Orica Limited through grant RG63368. Calculations were performed on the Darwin Supercomputer of the University of Cambridge High Performance Computing Service, using Strategic Research Infrastructure Funding from the Higher Education Funding Council for England, and funding from the Science and Technology Facilities Council.
[1] A. R. Koblitz, S. Lovett, and N. Nikiforakis, Phys. Rev. Fluids 3, 023301 (2018).

[2] K. Sverdrup, N. Nikiforakis, and A. Almgren, Phys. Fluids 30, 093102 (2018).

[3] L. Michael and N. Nikiforakis, J. Comp. Phys. 367, 1 (2018).

[4] S. Schoch, N. Nikiforakis, and B. J. Lee, Phys. Fluids 25, 086102 (2013).

[5] S. Schoch, N. Nikiforakis, B. J. Lee, and R. Saurel, Combust. Flame 160, 1883 (2013).

[6] S. Schoch, K. Nordin-Bates, and N. Nikiforakis, J. Comp.1 Phys. 252, 163 (2013).

[7] S. Eliezer, A. K. Ghatak, and H. Hora, Fundamentals of Equations of State (World Scientific, Singapore, 2002).
[8] R. Menikoff, Complete EOS for PBX 9502, Tech. Rep. LA-UR09-06529, Los Alamos National Laboratory, Los Alamos, NM, USA, 2009.

[9] O. Heuzé, C. R. Mec. 340, 679 (2012).

[10] R. Menikoff, JWL equation of state, Tech. Rep. LA-UR-1529536, Los Alamos National Laboratory, Los Alamos, NM, USA, 2015.

[11] S. D. Wilkinson, M. Braithwaite, N. Nikiforakis, and L. Michael, J. Appl. Phys. 122, 225112 (2017).

[12] P. F. Weck, K. R. Cochrane, S. Root, J. M. D. Lane, L. Shulenburger, J. H. Carpenter, T. Sjostrom, T. R. Mattsson, and T. J. Vogler, Phys. Rev. B 97, 125106 (2018).

[13] A. Rakhel, J.Phys. Condens. Matter 30, 295602 (2018). 
[14] M. L. Cohen, Phys. Scr. 1982, 5 (1982).

[15] D. C. Swift, G. J. Ackland, A. Hauer, and G. A. Kyrala, Phys. Rev. B 64, 214107 (2001).

[16] A. A. Correa, L. X. Benedict, M. A. Morales, P. A. Sterne, J. I. Castor, and E. Schwegler, arXiv:1806.01346.

[17] S. Hiermaier, Structures Under Crash and Impact (Springer, Berlin, 2008).

[18] S. Godunov and E. Romenskii, J. Appl. Mech. Tech. Phys. 13, 868 (1972).

[19] B. J. Plohr and D. H. Sharp, Adv. Appl. Math. 9, 481 (1988).

[20] G. Miller and P. Colella, J. Comput. Phys. 167, 131 (2001).

[21] P. Barton, D. Drikakis, E. Romenski, and V. Titarev, J. Comput. Phys. 228, 7046 (2009).

[22] E. F. Toro, Riemann Solvers and Numerical Methods for Fluid Dynamics: A Practical Introduction (Springer Science \& Business Media, Berlin, Heidelberg, 2013).

[23] D. S. Balsara and C.-W. Shu, J. Comput. Phys. 160, 405 (2000).

[24] J. M. Soler, E. Artacho, J. D. Gale, A. García, J. Junquera, P. Ordejón, and D. Sánchez-Portal, J. Phys.: Condens. Matter 14, 2745 (2002).

[25] J. P. Perdew, K. Burke, and M. Ernzerhof, Phys. Rev. Lett. 77, 3865 (1996).

[26] O. Strickson and E. Artacho, Phys. Rev. B 93, 094107 (2016).
[27] J. Moreno and J. M. Soler, Phys. Rev. B 45, 13891 (1992).

[28] J. Tersoff, Phys. Rev. Lett. 56, 632 (1986).

[29] A. Chentsov and P. Levashov, Contrib. Plasma Phys. 52, 33 (2012).

[30] L. Landau and E. Lifshitz, Statistical Physics, 3rd ed., Course of Theoretical Physics Vol. 5 (Elsevier Science, Amsterdam, 1980).

[31] D. MacKay, Information Theory, Inference and Learning Algorithms (Cambridge University Press, Cambridge, UK, 2003).

[32] C. Rasmussen and C. Williams, Gaussian Processes for Machine Learning, Adaptative Computation and Machine Learning Series (MIT Press, Cambridge, MA, 2006).

[33] T. Goto, T. Sato, and Y. Syono, Jpn. J. Appl. Phys. 21, L369 (1982).

[34] S. J. Turneaure and Y. Gupta, Appl. Phys. Lett. 90, 051905 (2007).

[35] T. W. Simpson, D. K. J. Lin, and W. Chen, Int. J. Reliab. Appl. 2, 209 (2001).

[36] O. T. Strickson, Ph.D. thesis, University of Cambridge, 2016, available at https://doi.org/10.17863/CAM.44845.

[37] J. Simo and T. Hughes, Computational Inelasticity, Interdisciplinary Applied Mathematics (Springer, New York, 2000).

[38] S. Chandra, M. Samal, V. Chavan, and S. Raghunathan, Int. J. Plast. 101, 188 (2018). 